\title{
METRIC BAUMGARTNER THEOREMS AND UNIVERSALITY
}

\author{
Stefan Geschke and Menachem Kojman
}

\begin{abstract}
It is consistent with the axioms of set theory that for every metric space $X$ which is isometric to some separable Banach space or to Urysohn's universal separable metric space $\mathbb{U}$ the following holds:

$(\star)_{X}$ There exists a nowhere meager subspace of $X$ of cardinality $\aleph_{1}$ and any two nowhere meager subsets of $X$ of cardinality $\aleph_{1}$ are almost isometric to each other.

As a corollary, it is consistent that the Continuum Hypothesis fails and the following hold:

(1) There exists an almost-isometry ultrahomogeneous and universal element in the class of separable metric spaces of size $\aleph_{1}$.

(2) For every separable Banach space $X$ there exists an almost-isometry conditionally ultrahomogeneous and universal element in the class of subspaces of $X$ of size $\aleph_{1}$.

(3) For every finite dimensional Banach space $X$, there is a unique universal element up to almost-isometry in the class of subspaces of $X$ of size $\aleph_{1}$
\end{abstract}

\section{Introduction}

In this paper we deal with metric similarity, metric uniqueness and metric universality in models of set theory that violate the Continuum Hypothesis $(\mathrm{CH})$. Originally motivated by questions about metric universality, we prove the consistency with the usual axioms of set theory of a strong statement about the homogeneity of separable Banach spaces and of Urysohn's universal separable metric space.

Let $(X, d)$ be the underlying metric structure of some separable Banach space, and consider the following problem: suppose $A \subseteq X$ satisfies that $A \cap U$ is of the second category in $U$ for every nonempty open $U \subseteq X$. Can one find a set $B \subseteq X$ of the same cardinality as $A$, satisfying the same condition as $A$, such that $B$ is not in the autohomeomorphism group orbit of $A$ ?

If one assumes the Continuum Hypothesis, then such a $B$ is very easily found: by $\mathrm{CH}$ the cardinality of $A$ is necessarily equal to $|X|$ and either $X$ itself of $X \backslash H$, where $H$ is a hyperplane of $X$, is not homeomorphic to $A$.

The main result below shows that without appealing to an additional axiom, like $\mathrm{CH}$, finding $B$ is impossible, because it is consistent that for every separable Banach space $X$ and two nowhere meager sets $A, B \subseteq X$ with $|A|=|B|=\aleph_{1}$ not only that $A$ can be carried over onto $B$ by an autohomeomorphism of $X$, it actually holds that $A$ maps onto $B$ by autohomeomorphisms of $X$ which are arbitrarily close to being isometries, namely are bi-Lipschitz with Lipschitz constants arbitrarily close to 1 .

Received by the editors May 5, 2006.

2000 Mathematics Subject Classification. Primary: 54E40, 51M04, 03E35; Secondary: 46B20.

Key words and phrases. Metric space, almost-isometry, almost-isometric embedding, Urysohn's space, Oracle Forcing, Universality. 
To present better the picture at large, we begin by describing the subject in the related setting of linearly ordered sets. The reader may consult the introduction to [8] for additional introductory material.

1.1. Order isomorphism and universality. Up to order isomorphism there is a unique countable dense subset of $\mathbb{R}$. The ordertype of such a set is the unique ultrahomogeneous and universal member in the class of countable linearly ordered sets. Consequently, $\mathbb{R}$ is universal in the class of separable linearly ordered sets and its ordertype is determined uniquely as the completion of the countable ultrahomogeneous and universal ordering. These results were proved of course by Cantor. If one does not assume completeness, then by Sierpinski [15], there are $2^{2^{\aleph_{0}}}$ continuum dense subsets of $\mathbb{R}$ that are pairwise order incomparable.

Under $\mathrm{CH}, \kappa$-dense subsets of $\mathbb{R}$ exist only for $\kappa \in\left\{\aleph_{0}, 2^{\aleph_{0}}\right\}$. But as is well-known since Cohen's invention of forcing, also the negation of the Continuum Hypothesis is consistent with the axioms of set theory - in fact, the continuum may be arbitrarily large. Thus, for every cardinal $\kappa>\aleph_{0}$ it is meaningful to inquire the consistency with the axioms of the statement, and of the negation of the statement, that $2^{\aleph_{0}}>\kappa$ and all $\kappa$-dense subsets of $\mathbb{R}$ are order-isomorphic to each other. The same applies to the statement of the existence of a universal separable linearly ordered set of cardinality $\kappa$, which, of course, follows from the previous one.

In Cohen extensions, as well as in in Solovay's random real extensions, with arbitrary large continuum, neither of these statements holds for any $\kappa, \aleph_{0}<\kappa<2^{\aleph_{0}}$ (see $[7,8]$ ). Thus, the negations of these two statements (for an arbitrary $\kappa>\aleph_{0}$ ) are easily consistent, being valid in standard models for the negation of $\mathrm{CH}$.

Positive consistency was somewhat harder. Baumgartner, in a classic paper in set theory [3], proved the consistency of $2^{\aleph_{0}}=\aleph_{2}$ and any two $\aleph_{1}$-dense subsets of $\mathbb{R}$ are order isomorphic to each other. Baumgartner's method necessitated the validity of $\mathrm{CH}$ in intermediate models of his forcing iteration, and its novelty at the time was further supported by the fact that the argument could not be easily lifted to $\aleph_{2}$. In fact, the consistency of Baumgartner's statement for $\aleph_{2}$-dense sets is still open.

Abraham and Shelah have shown that Baumgartner's result did not follow from Martin's Axiom [2]. Abraham, Rubin and Shelah have investigated many variants of Baumgartner's result and have also proved that Baumgartner's statement for $\aleph_{1^{-}}$ dense sets was consistent with continuum larger than $\aleph_{2}[1]$. Later it was observed that Baumgartner's result followed from the Proper Forcing Axiom [4], but this gave no information for $\kappa>\aleph_{1}$, as PFA was later shown to imply that $2^{\aleph_{0}}=\aleph_{2}$ (see [16] and $[17])$.

Recently it was shown by Moore [11] that PFA also implies Shelah's Basis Conjecture for linear orders, i.e., there are five uncountable linear orders such that every uncountable linear order contains a copy of one of the five.

The problem of universality at $\aleph_{1}$ in the class of all (rather than only separable) linearly ordered sets has also been investigated. If $\mathrm{CH}$ holds, then there is a saturated linear order (unique for that property) of cardinality $\aleph_{1}$, which is also universal. However, since the existence of a universal linearly ordered set of cardinality $\aleph_{1}$ implies the existence of a universal separable one, in Cohen or Solovay extensions there are no universal linearly ordered sets of cardinality $\aleph_{1}$. 
It took a remarkable development in forcing technique to settle the consistency of a universal linear ordering in $\aleph_{1}<2^{\aleph_{0}}$. In [12] Shelah introduced both his Proper Forcing and his Oracle Forcing methods, and used them to prove the consistency of $2^{\aleph_{0}}=\aleph_{2}$ with the existence a universal linearly ordered set of cardinality $\aleph_{1}$. The singularity and difficulty of this result are appreciated adequately in the light of a much later result by Kojman and Shelah [7]: $\aleph_{1}$ is the unique uncountable regular cardinal for which this consistency holds; for every regular $\kappa>\aleph_{1}$ the inequality $2^{\aleph_{0}}>\kappa$ implies that fewer than $2^{\aleph_{0}}$ linearly ordered sets, each of cardinality $\kappa$, do not suffice to order-embed all linearly ordered sets of cardinality $\kappa$. (For a recent survey of applications of the combinatorial method introduced in this proof see [6].)

1.2. Metric isomorphisms and universality. Let us now turn to metric similarity and embeddability. Kojman and Shelah have introduced the notions of almostisometry and almost-isometric embedding between metric spaces and were able to obtain with respect to these relations several of the results described above in the setting of linearly ordered sets. Up to almost-isometry there is a unique countable dense subset of $\mathbb{R}$, which is the unique ultrahomogeneous and universal member in the class of linear countable metric spaces for almost-isometric embeddings. More importantly, up to almost-isometry there is a unique countable dense subset of Urysohn's universal separable metric space $\mathbb{U}$ that is the unique almost-isometry ultrahomogeneous and universal member in the class of countable metric spaces. In fact, the Urysohn space is characterized as the completion of such a countable space. In Cohen or Solovay extensions both almost-isometric uniqueness of $\kappa$-dense subsets - of $\mathbb{R}$ as well as of $\mathbb{U}$ - and the existence of an almost-isometry universal metric space of size $\kappa$ fail for all $\kappa$ with $\aleph_{0}<\kappa<2^{\aleph_{0}}$. Furthermore, if $\kappa>\aleph_{1}$ is a regular cardinal and $2^{\aleph_{0}}>\kappa$, then fewer than $2^{\aleph_{0}}$ metric spaces (not necessarily separable) of cardinality $\kappa$ do not suffice to almost isometrically embed all metric spaces of cardinality $\kappa$. This result leaves $\aleph_{1}$ as the only regular cardinal at which the existence of an almost-isometry universal space below the continuum may be consistent. This consistency is not known.

In the class of separable metric spaces also positive results were proved in [8]. It is consistent that fewer than continuum separable metric spaces of size $\kappa$ almost isometrically embed all separable metric spaces of size $\kappa$, for regular $\kappa \in\left(\aleph_{0}, 2^{\aleph_{0}}\right)$. Thus, for $\kappa=\aleph_{2}<2^{\aleph_{0}}$, it may require only a small collection of separable metric spaces on $\aleph_{2}$ to almost-isometrically embed all others, but it always takes a larger number of general metric spaces on $\aleph_{2}$ for the similar task. For $\kappa=\aleph_{1}$ this result approximated the consistency of a universal separable metric space in $\aleph_{1}<2^{\aleph_{0}}$, but left it open.

Finally, it was shown in [8] that the almost-isometry analog of Baumgartner's result was simply false: in every "reasonable" metric space (see Section 2 below; we only care here that $\mathbb{R}$ and $\mathbb{U}$ are "reasonable") there are many pairwise almost-isometry incomparable $\aleph_{1}$-dense subsets. This seemed at the time to block the approach to universality via Baumgartner type uniqueness.

Yet, a metric Baumgartner theorem is exactly what we prove here (for $\mathbb{R}, \mathbb{U}$ and every separable Banach space) among whose consequences is the consistency of an almost-isometry universal separable metric space of cardinality $\aleph_{1}<2^{\aleph_{0}}$. We use Shelah's Oracle Forcing method from [12] (also see [14, Chapter IV]) to prove Baumgartner's metric analog with an additional, topological condition: any two subsets of 
$\mathbb{U}$ or any separable Banach space that are nowhere meager, i.e., non-meager in any open subset, and of size $\aleph_{1}$ are almost isometric to each other (Theorem 5.1).

It is worth pointing out that almost isometries between dense subsets of a complete metric space extend to the whole space. Thus, we obtain the consistency of a statement saying that the spaces under consideration are very homogeneous.

\section{Notation and preliminaries}

Say that two metric spaces $\left(X, d_{X}\right)$ and $\left(Y, d_{Y}\right)$ are almost isometric if for every real constant $K>1$ there is a homeomorphism $f: X \rightarrow Y$ that satisfies

$$
d_{X}\left(x_{1}, x_{2}\right) / K<d_{Y}\left(f\left(x_{1}\right), f\left(x_{2}\right)\right)<K d_{X}\left(x_{1}, x_{2}\right) .
$$

That is, for every $K>1$ there exists a $K$-bi-Lipschitz homeomorphism between $X$ and $Y$.

We quote from [8]:

Theorem 2.1. Suppose $X$ is a separable metric space and there exists a constant $K>1$ so that for every open subset $U \subseteq X$ there is a $K$-bi-Lipschitz embedding of a non-empty open interval from the standard Cantor set into $U$. Then there are $2^{\aleph_{0}}$ pairwise bi-Lipschitz incomparable $\aleph_{1}$-dense subsets of $X$.

Let $X$ be a perfect and complete metric space. Let $(\star)_{X}$ be the following statement about $X$ :

$(\star)_{X}$ There exists a nowhere meager subset of $X$ of cardinality $\aleph_{1}$ and any two nowhere meager subsets of $X$ of size $\aleph_{1}$ are almost isometric to each other.

Fact 2.2. If $(\star)_{X}$ holds for any separable metric space that contains an uncountable nowhere dense set, then

$$
2^{\aleph_{0}}=2^{\aleph_{1}} .
$$

In particular, this equality follows from $(\star)_{\mathbb{R}}$.

Proof. Let $Z$ be an uncountable nowhere dense subset of $X$. We may assume that $Z$ is of size $\aleph_{1}$. By $(\star)_{X}$, there is a nowhere meager subset $Y$ of $X$ of cardinality $\aleph_{1}$. After subtracting $Z$ from $Y$ we may assume that $Y$ and $Z$ are disjoint. For every $W \subseteq Z$ let $Y_{W}=Y \cup W$. This gives a collection of $2^{\aleph_{1}}$ nowhere meager subsets of $X$, each of cardinality $\aleph_{1}$.

By $(\star)_{X}$, for each $W \subseteq Z$ we may fix $f_{W}: Y \rightarrow Y_{W}$, a bi-Lipschitz homeomorphism. Each $f_{W}$ extends to an autohomeomorphism $\bar{f}_{W}$ of the completion $\bar{X}$ of $X$. However, being separable, $\bar{X}$ has only $2^{\aleph_{0}}$ autohomeomorphisms. Hence $2^{\aleph_{1}}=2^{\aleph_{0}}$.

We shall prove below the consistency of $2^{\aleph_{0}}=\aleph_{2}$ together with $(\star)_{X}$ for every metric space $X$ that is isometric to some separable Banach space or to Urysohn's universal separable space $\mathbb{U}$, and discuss some of the consequences of this consistency in universality theory.

A metric space $X$ is almost-isometry universal for a class of metric spaces if for every space $Y$ in the class and every $K>1$ there exists a $K$-bi-Lipschitz embedding of $Y$ into $X$, and $X$ is almost-isometry ultrahomogeneous if for every $K>1$, every finite $K$-bi-Lipschitz map from $X$ to $X$ extends to a $K$-bi-Lipschitz autohomeomorphism of 
$X$. A subspace $Y \subseteq X$ is conditionally almost-isometry ultrahomogeneous if for every $K>1$ every finite $K$-bi-Lipschitz map from $Y$ to $Y$ which extends to a $K$-bi-Lipschitz autohomeomorphism of $X$ extends to a $K$-bi-Lipschitz autohomeomorphism of $Y$

\section{Oracle forcing}

We quote some definitions and results about Shelah's oracle forcing from Shelah's [12]. Some readers may prefer the more detailed presentation of oracle forcing in chapter IV of [14].

Definition 3.1. A sequence $\mathbf{S}=\left(\mathcal{S}_{\alpha}\right)_{\alpha<\omega}$ of countable transitive models of ZFC except the power set axiom is called an $\aleph_{1}$-oracle if for all $A \subseteq \omega_{1}$, the set $\left\{\alpha<\omega_{1}\right.$ : $\left.A \cap \alpha \in \mathcal{S}_{\alpha}\right\}$ is stationary in $\omega_{1}$.

Note that if $\diamond_{\aleph_{1}}$ holds, then there is an $\aleph_{1}$-oracle.

Definition 3.2. Let $\mathbf{S}$ be an $\aleph_{1}$-oracle. A forcing notion $\mathbb{P}$ satisfies the $\mathbf{S}$-chain condition, if for all $\mathbb{P}^{\prime} \subseteq \mathbb{P}$ of size $\leq \aleph_{1}$ there is $\mathbb{P}^{\prime \prime} \subseteq \mathbb{P}$ also of size $\leq \aleph_{1}$, a 1-1 function $f: \mathbb{P}^{\prime \prime} \rightarrow \omega_{1}$ and a set $A \subseteq \omega_{1}$ such that $\mathbb{P}^{\prime} \subseteq \mathbb{P}^{\prime \prime}$ and for all limit ordinals $\alpha<\omega_{1}$, if $A \cap \alpha \in \mathcal{S}_{\alpha}, S \in \mathcal{S}_{\alpha} \cap \mathcal{P}(\alpha)$ and $f^{-1}[S]$ is predense in $f^{-1}[\alpha]$, then $f^{-1}[S]$ is predense in $\mathbb{P}$.

The following Lemma says that forcing notions which satisfy an oracle chain condition can be iterated.

Lemma 3.3 (Shelah [12]). Let $\mathbf{S}$ be an $\aleph_{1}$-oracle.

(a) Suppose that $\mathbb{P}$ is a forcing notion satisfying the $\mathbf{S}$-chain condition. Then there is a $\mathbb{P}$-name $\dot{\mathbf{T}}$ for an $\aleph_{1}$-oracle such that for every $\mathbb{P}$-name $\dot{\mathbb{Q}}$ for a forcing notion the following holds: If

$$
\Vdash_{\mathbb{P}} \text { “过 satisfies the } \dot{\mathbf{T}} \text {-chain condition”, }
$$

then $\mathbb{P} * \dot{\mathbb{Q}}$ satisfies the $\mathbf{S}$-chain condition.

(b) If for some ordinal $\delta,\left(\mathbb{P}_{\alpha}\right)_{\alpha<\delta}$ is a continuous increasing chain of forcing notions satisfying the $\mathbf{S}$-chain condition, then the direct limit $\bigcup_{\alpha<\delta} \mathbb{P}_{\alpha}$ satisfies the S-chain condition.

The main property of the oracle chain condition which we require is that forcing notions satisfying the oracle chain condition preserve the non-meagerness of the set of ground model reals.

Lemma 3.4 (Shelah [12]). Assume there is an $\aleph_{1}$-oracle $\mathbf{S}^{\prime}$. Then there is an $\aleph_{1}$ oracle $\mathbf{S}$ such that for every forcing notion $\mathbb{P}$ satisfying the $\mathbf{S}$-chain condition and every $\mathbb{P}$-generic filter $G$ over the ground model $V$, in $V[G]$ the set $\mathbb{R} \cap V$ is non-meager.

\section{Forcing bi-Lipschitz bijections}

We wish to add sufficiently bi-Lipschitz bijections between two large subsets of a separable Banach space by forcing. The following lemma says that we will not run into trouble at a finite stage of the construction. 
Lemma 4.1. Let $B$ be a Banach space and $K_{0}>1$. Suppose $p$ is a finite partial function from $B$ to $B$ that extends to a $K_{0}$-bi-Lipschitz bijection $f: B \rightarrow B$. Then for every $x_{0} \in B \backslash \operatorname{dom}(p)$ and every $K_{1}>K_{0}$ there is a nonempty open set $O \subseteq B$ such that for all $y \in O, p \cup\left\{\left(x_{0}, y\right)\right\}$ extends to a $K_{1}$-bi-Lipschitz bijection from $B$ to $B$.

Proof. Let $x_{0} \in B \backslash \operatorname{dom}(p)$. We may assume that $\operatorname{dom}(p)$ is non-empty. Let $\delta=$ $\min \left\{\left\|x_{0}-x\right\|: x \in \operatorname{dom}(p)\right\}$ and let $y_{0}=f\left(x_{0}\right)$. Since $K_{0}<K_{1}$ there is $c>0$ such that

$$
K_{0}+c<K_{1} \quad \text { and } \quad \frac{1}{K_{0}}-c>\frac{1}{K_{1}} .
$$

Let

$$
O=\left\{y \in B: \frac{\left\|y-y_{0}\right\|}{\delta}<c\right\} .
$$

For every $x \in B$ and $y \in O$ let

$$
g(x)=\left\{\begin{array}{l}
0, \text { if }\left\|x-x_{0}\right\|>\delta \text { and } \\
\left(y-y_{0}\right) \cdot\left(1-\frac{\left\|x-x_{0}\right\|}{\delta}\right), \text { if }\left\|x-x_{0}\right\| \leq \delta .
\end{array}\right.
$$

It is easily checked that $g: B \rightarrow B$ is Lipschitz of constant $c$. Clearly, the function $f+g: B \rightarrow B$ extends $p \cup\left\{\left(x_{0}, y\right)\right\}$.

We have to check that $f+g$ is a $K_{1}$-bi-Lipschitz bijection. Let $x, x^{\prime}$ be distinct elements of $B$. Then by the choice of $c$,

$$
\frac{\left\|(f+g)(x)-(f+g)\left(x^{\prime}\right)\right\|}{\left\|x-x^{\prime}\right\|} \leq \frac{\left\|f(x)-f\left(x^{\prime}\right)\right\|+\left\|g(x)-g\left(x^{\prime}\right)\right\|}{\left\|x-x^{\prime}\right\|}<K_{0}+c<K_{1}
$$

On the other hand,

$$
\frac{\left\|(f+g)(x)-(f+g)\left(x^{\prime}\right)\right\|}{\left\|x-x^{\prime}\right\|} \geq \frac{\left\|f(x)-f\left(x^{\prime}\right)\right\|-\left\|g(x)-g\left(x^{\prime}\right)\right\|}{\left\|x-x^{\prime}\right\|}>\frac{1}{K_{0}}-c>\frac{1}{K_{1}},
$$

again by the choice of $c$.

To see that $f+g$ is onto let $z_{0} \in B$ and consider the map

$$
h: B \rightarrow B ; x \mapsto f^{-1}\left(z_{0}-g(x)\right) .
$$

By the choice of $c, \frac{1}{K_{0}}-c>0$ and therefore $K_{0} \cdot c<1$. Since $f^{-1}$ is Lipschitz of constant $K_{0}$ and $g$ is Lipschitz of constant $c, h$ is a contraction. Therefore, $h$ has a fixed point $x$. Now

$$
(f+g)(x)=f(h(x))+g(x)=f\left(f^{-1}\left(z_{0}-g(x)\right)\right)+g(x)=z_{0} .
$$

In other words, $z_{0}$ is in the range of $f+g$.

Lemma 4.2. Let $\mathbf{S}$ be an $\aleph_{1}$-oracle and $K>1$. Suppose $X$ and $Y$ are nowhere meager subsets of size $\aleph_{1}$ of a separable Banach space $B$. Then there is a forcing notion $\mathbb{P}$ of size $\aleph_{1}$ satisfying the $\mathbf{S}$-chain condition such that

$$
\Vdash_{\mathbb{P}} \text { "There is a K-bi-Lipschitz bijection between } X \text { and } Y \text { ". }
$$


Proof. We will force by finite approximations of $K$-bi-Lipschitz bijections from $B$ to $B$. A finite partial function $p$ from $B$ to $B$ is a potential condition if for some $K^{\prime} \in(1, K), p$ extends to a $K^{\prime}$-bi-Lipschitz bijection from $B$ to $B$.

By recursion on $\alpha<\omega_{1}$ we will construct sequences $\left(X_{\alpha}\right)_{\alpha<\omega}$ and $\left(Y_{\alpha}\right)_{\alpha<\omega_{1}}$ of subsets of $X$, respectively $Y$, a sequence $\left(\mathbb{P}_{\alpha}\right)_{\alpha \leq \omega_{1}}$ of forcing notions and a sequence $\left(f_{\alpha}\right)_{\alpha<\omega_{1}}$ of functions such that the following conditions are satisfied:

(1) Every $X_{\alpha}$ is a countable dense subset of $X$ and every $Y_{\alpha}$ is a countable dense subset of $Y$.

(2) The sets $X_{\alpha}$ are pairwise disjoint and so are the sets $Y_{\alpha}$. Moreover, $X=$ $\bigcup_{\alpha<\omega_{1}} X_{\alpha}$ and $Y=\bigcup_{\alpha<\omega_{1}} Y_{\alpha}$.

(3) For sequences $\left(A_{\gamma}^{0}\right)_{\gamma<\delta}$ and $\left(A_{\gamma}^{1}\right)_{\gamma<\delta}$ of subsets of $B$ let $\mathbb{P}\left(\left(A_{\gamma}^{0}\right)_{\gamma<\delta},\left(A_{\gamma}^{1}\right)_{\gamma<\delta}\right)$ be the set of finite partial functions $p$ from $\bigcup_{\gamma<\delta} A_{\gamma}^{0}$ to $\bigcup_{\gamma<\delta} A_{\gamma}^{1}$ that are potential conditions such that for all $x \in \operatorname{dom}(p)$ and all $\gamma<\alpha$,

$$
x \in A_{\gamma}^{0} \quad \Leftrightarrow \quad p(x) \in A_{\gamma}^{1} .
$$

$\mathbb{P}\left(\left(A_{\gamma}^{0}\right)_{\gamma<\delta},\left(A_{\gamma}^{1}\right)_{\gamma<\delta}\right)$ is ordered by reverse inclusion.

Then $\mathbb{P}^{\alpha}=\mathbb{P}\left(\left(X_{\gamma}\right)_{\gamma<\alpha},\left(Y_{\gamma}\right)_{\gamma<\alpha}\right)$.

(4) For every $\alpha<\omega_{1}$ with $\alpha>0, f_{\alpha}$ is a bijection from $\mathbb{P}^{\alpha}$ onto $\omega \cdot \alpha$. If $\alpha<\beta<\omega_{1}$, then $f_{\beta}$ is an extension of $f_{\alpha}$.

(5) For all $\alpha<\omega_{1}$, if for some $\beta \leq \alpha$ and some $S \in \mathcal{S}_{\beta}, f_{\beta}^{-1}[S]$ is predense in $\mathbb{P}^{\beta}$, then every condition in $\mathbb{P}^{\alpha+1}$ is compatible (in $\mathbb{P}^{\alpha+1}$ ) with an element of $f_{\beta}^{-1}[S]$, i.e., $f_{\beta}^{-1}[S]$ is predense in $\mathbb{P}^{\alpha+1}$.

Suppose the recursion can be carried out. We claim that $\mathbb{P}=\mathbb{P}^{\omega_{1}}$ works for the lemma.

Let $G$ be $\mathbb{P}$-generic over the ground model. Then clearly, $b=\bigcup G$ is a $K$-biLipschitz mapping from a subset of $X$ to a subset of $Y$. We have to check that $\operatorname{dom}(b)=X$ and $\operatorname{ran}(b)=Y$. We only give the argument for $\operatorname{dom}(b)=X$. The proof of $\operatorname{ran}(Y)=Y$ is symmetric.

Let $x$ be a point in $X$ and let $p$ be a condition in $\mathbb{P}$. Suppose that $x \notin \operatorname{dom}(p)$. Let $\alpha<\omega_{1}$ be such that $x \in X_{\alpha}$. By Lemma 4.1, there is an open set $O \subseteq \mathbb{R}$ such that for all $y \in O, p \cup\{(x, y)\}$ is a potential condition.

Since $Y_{\alpha}$ is dense in $Y$ and $Y$ is dense in $B$, there is $y \in O \cap Y_{\alpha}$. Now $p \cup\{(x, y)\} \in \mathbb{P}$ extends $p$ and is again a condition in $\mathbb{P}$. It follows that the set of conditions that have $x$ in their domain is dense in $\mathbb{P}$. So by genericity, $x \in \operatorname{dom}(b)$.

We now show that $f=\bigcup_{\alpha<\omega_{1}} f_{\alpha}$ witnesses the $\mathbf{S}$-chain condition of $\mathbb{P}$.

Let $\alpha$ be a limit ordinal below $\omega_{1}$. Then for some $\beta<\omega_{1}, \alpha=\omega \cdot \beta$ and hence, by (4), $f^{-1}[\alpha]=\mathbb{P}^{\beta}$. Suppose that for some $S \in \mathcal{S}_{\alpha}, f^{-1}[S]$ is predense in $f^{-1}[\alpha]=\mathbb{P}^{\beta}$. Then, by $(5), f^{-1}[S]$ is predense in $\mathbb{P}^{\gamma+1}$ for every $\gamma<\omega_{1}$ with $\gamma \geq \beta$. Hence $f^{-1}[S]$ is predense in $\mathbb{P}$.

Let us turn to the recursive construction. For $Z \in\{X, Y\}$ let $\prec_{Z}$ be a wellordering on $Z$ of order type $\omega_{1}$. Let $X_{0}$ and $Y_{0}$ be countable dense subsets of $X$, respectively $Y$. Suppose for some $\alpha<\omega_{1}$ we have constructed $\left(X_{\gamma}\right)_{\gamma<\alpha},\left(Y_{\gamma}\right)_{\gamma<\alpha}$ and $f_{\alpha}$.

By recursion on $k<\omega$ we will define sequences $\left(x_{k}\right)_{k<\omega}$ and $\left(y_{k}\right)_{k<\omega}$ and then put $X_{\alpha}=\left\{x_{k}: k<\omega\right\}$ and $Y_{\alpha}=\left\{y_{k}: k<\omega\right\}$. Extending $f_{\alpha}$ to $f_{\alpha+1}$ is straight forward, so we will not mention it anymore. 
Fix an enumeration $\left(O_{n}\right)_{n \in \omega}$ of all nonempty members of some countable basis for the topology on $B$. To make sure that $X_{\alpha}$ and $Y_{\alpha}$ are dense, for all $k>0$ we will choose $x_{k} \in X \cap O_{k}$ and $y_{k} \in Y \cap O_{k}$.

Suppose $\alpha$ is an even ordinal. Then let $x_{0}$ be the $\prec_{X}$-minimal element of $X \backslash$

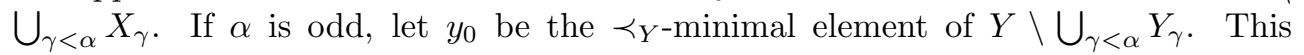
guarantees that in the end we have $X=\bigcup_{\gamma<\omega_{1}} X_{\gamma}$ and $Y=\bigcup_{\gamma<\omega_{1}} Y_{\gamma}$.

If $\alpha$ is even, then we already know $x_{0}$ and choose $y_{0}$ next, then $x_{1}$, then $y_{1}$ and so on. If $\alpha$ is odd, then we already know $y_{0}$ and choose $x_{0}$ next, then $y_{1}$, then $x_{1}$ and so on.

To make sure that (5) is satisfied for $\alpha$, we will choose $\left(x_{k}\right)_{k<\omega}$ and $\left(y_{k}\right)_{k<\omega}$ so that for even $\alpha$ it holds that

(even) for all $k<\omega,(5)$ is true for $\mathbb{P}\left(\left(X_{\gamma}\right)_{\gamma<\alpha} \frown\left\{x_{l}: l<k\right\},\left(Y_{\gamma}\right)_{\gamma<\alpha} \frown\left\{y_{l}: l<k\right\}\right)$ and $\mathbb{P}\left(\left(X_{\gamma}\right)_{\gamma<\alpha} \frown\left\{x_{l}: l \leq k\right\},\left(Y_{\gamma}\right)_{\gamma<\alpha} \frown\left\{y_{l}: l<k\right\}\right)$ instead of $\mathbb{P}^{\alpha+1}$

and so that for odd $\alpha$ it holds that

(odd) for all $k<\omega,(5)$ is true for $\mathbb{P}\left(\left(X_{\gamma}\right)_{\gamma<\alpha} \frown\left\{x_{l}: l<k\right\},\left(Y_{\gamma}\right)_{\gamma<\alpha} \frown\left\{y_{l}: l<k\right\}\right)$ and $\mathbb{P}\left(\left(X_{\gamma}\right)_{\gamma<\alpha} \frown\left\{x_{l}: l<k\right\},\left(Y_{\gamma}\right)_{\gamma<\alpha} \frown\left\{y_{l}: l \leq k\right\}\right)$ instead of $\mathbb{P}^{\alpha+1}$.

We concentrate on one case, namely that $\alpha$ is odd and we have already defined $\left(x_{l}\right)_{l<k}$ and $\left(y_{l}\right)_{l<k}$ for some $k>0$, and we are now looking for $y_{k}$. The other three cases are symmetric.

In order to satisfy (odd), $y_{k}$ has to meet countably many constraints. Namely, whenever $\beta \leq \alpha$ is a limit ordinal, $S \in \mathcal{S}_{\beta}, f_{\beta}^{-1}[S]$ is predense in $\mathbb{P}^{\beta}$ and $p \in$ $\mathbb{P}\left(\left(X_{\gamma}\right)_{\gamma<\alpha} \frown\left\{x_{l}: l<k\right\},\left(Y_{\gamma}\right)_{\gamma<\alpha} \frown\left\{y_{l}: l \leq k\right\}\right)$ uses $y_{k}$, then $p$ is compatible with some element of $f_{\beta}^{-1}[S]$. We will show that for each constraint, the set of $y \in Y$ satisfying it is open and dense. Since $Y$ is nowhere meager, there is $y \in$ $O_{k-1} \cap Y \backslash\left(\bigcup_{\gamma<\alpha} Y_{\gamma} \cup\left\{y_{l}: l<k\right\}\right)$ satisfying all these constraints. Putting $y_{k}=y$ ensures (odd).

We finish the proof of the lemma by showing that the set of $y$ meeting a single constraint is open and dense.

Let $p \in \mathbb{P}\left(\left(X_{\gamma}\right)_{\gamma<\alpha} \frown\left\{x_{l}: l<k\right\},\left(Y_{\gamma}\right)_{\gamma<\alpha} \frown\left\{y_{l}: l<k\right\}\right)$ and $\beta \leq \alpha$. Suppose that $x \in\left\{x_{l}: l<k\right\}$ is not in $\operatorname{dom}(p)$. Suppose further that $S \in \mathcal{S}_{\beta}$ is such that $f_{\beta}^{-1}[S]$ is predense in $\mathbb{P}^{\beta}$. Finally, let $O$ be a non-empty open subset of $B$. We show that there is a non-empty open set $U \subseteq O$ such that for all $y \in U$, if

$$
p \cup\{(x, y)\} \in \mathbb{P}\left(\left(X_{\gamma}\right)_{\gamma<\alpha} \frown\left\{x_{l}: l<k\right\},\left(Y_{\gamma}\right)_{\gamma<\alpha} \frown\left(\left\{y_{l}: l<k\right\} \cup\{y\}\right)\right),
$$

then in that partial order, $p \cup\{(x, y)\}$ is compatible with some element of $f_{\beta}^{-1}[S]$.

If there is no $y \in O$ such that $p \cup\{(x, y)\}$ is a potential condition, then we can choose $U=O$. Now suppose that for some $y \in O, p \cup\{(x, y)\}$ is a potential condition. By Lemma 4.1, there is $c>0$ such that for all $y^{\prime} \in \mathbb{R}$ with $\left|y-y^{\prime}\right|<c, y^{\prime} \in O$ and $p \cup\left\{\left(x, y^{\prime}\right)\right\}$ is a potential condition.

By the density of $X_{0}$ and $Y_{0}$ and by Lemma 4.1, there are $a \in X_{0}$ and $b \in Y_{0}$ such that $\|a-x\|<\frac{c}{2 K}$ and $p \cup\{(a, b),(x, y)\}$ is a potential condition. Now

$$
p \cup\{(a, b)\} \in \mathbb{P}\left(\left(X_{\gamma}\right)_{\gamma<\alpha} \frown\left\{x_{l}: l<k\right\},\left(Y_{\gamma}\right)_{\gamma<\alpha} \frown\left(\left\{y_{l}: l<k\right\}\right)\right)
$$

and for all $y^{\prime}$ such that $p \cup\left\{(a, b),\left(x, y^{\prime}\right)\right\}$ is a potential condition, $\left\|y^{\prime}-b\right\|<\frac{c}{2}$ and by $\|y-b\|<\frac{c}{2},\left\|y^{\prime}-y\right\|<c$. In particular, whenever $p \cup\left\{(a, b),\left(x, y^{\prime}\right)\right\}$ is a potential condition, then $y^{\prime} \in O$. This argument shows that by passing to a stronger condition 
if necessary, we may assume that all $y^{\prime}$ such that $p \cup\left\{\left(x, y^{\prime}\right)\right\}$ is a potential condition are elements of $O$.

By (5), $p$ is compatible with some condition $q \in f_{\beta}^{-1}[S]$. In particular, $p \cup q$ is a potential condition. By Lemma 4.1, there is an open set $U$ such that for all $y^{\prime} \in U$, $p \cup q \cup\left\{\left(x, y^{\prime}\right)\right\}$ is still a potential condition. By our assumption on $p, U \subseteq O$.

Lemma 4.2 remains true if the separable Banach space $B$ is replaced by the Urysohn space $\mathbb{U}$.

Lemma 4.3. Let $\mathbf{S}$ be an $\aleph_{1}$-oracle and $K>1$. Suppose $X$ and $Y$ are nowhere meager subsets of size $\aleph_{1}$ of the Urysohn space $\mathbb{U}$. Then there is a forcing notion $\mathbb{P}$ of size $\aleph_{1}$ satisfying the $\mathbf{S}$-chain condition such that

$$
\Vdash_{\mathbb{P}} \text { "There is an K-bi-Lipschitz bijection between } X \text { and } Y \text { ". }
$$

Proof. By Claim 16 in [8], $\mathbb{U}$ is almost-isometry ultrahomogeneous. This implies the analog of Lemma 4.1 for the Urysohn space easily. Now the proof of Lemma 4.3 is the same as the proof of Lemma 4.2

Lemma 4.4. If $(\star)_{\mathbb{U}}$ holds, then every nowhere meager subset $A \subseteq \mathbb{U}$ of size $\aleph_{1}$ is almost-isometry ultrahomogeneous.

If $X$ is a separable Banach space and $(\star)_{X}$ holds, then every nowhere meager subspace $A \subseteq X$ of cardinality $\aleph_{1}$ is conditionally almost-isometry ultrahomogeneous, namely, for every $K>1$, every finite $K$-bi-Lipschitz map from $A$ to $A$ extends to a $K$-bi-Lipschitz autohomeomorphism of $A$ - provided it extends to a K-bi-Lipschitz autohomeomorphism of $X$.

Proof. Since almost-isometry ultrahomogeneity is preserved under almost-isometry, it suffices to prove that there exists at least one almost-isometry ultrahomogeneous nowhere meager subset of $\mathbb{U}$ whose cardinality is $\aleph_{1}$. Since $\mathbb{U}$ is almost-isometry ultrahomogeneous, any subspace $A \subseteq \mathbb{U}$ can be enlarged to an almost-isometry ultrahomogeneous subspace of the same cardinality by a standard closure under bi-Lipschitz autohomeomorphisms argument (using only rational $K>1$ as constants).

The proof of the second clause in the Lemma is similar.

\section{The results}

Theorem 5.1. If ZFC is consistent, then so is $\mathrm{ZFC}+2^{\aleph_{0}}=\aleph_{2}+$ "for every metric space $X$, if $X$ is isometric to the Urysohn space or to some separable Banach space, then $(\star)_{X}$ holds", where $(\star)_{X}$ is the statement

$X$ has a nowhere meager subset of size $\aleph_{1}$ and any two nowhere meager subsets of $X$ of size $\aleph_{1}$ are almost isometric to each other.

Proof. We start from a model of $\diamond_{\aleph_{1}}+2^{\aleph_{1}}=\aleph_{2}$. In this model there is an $\aleph_{1}$-oracle $\mathbf{S}$ as in Lemma 3.4. We then perform a finite support iteration $\left(\left(\mathbb{P}_{\alpha}\right)_{\alpha \leq \omega_{2}},\left(\dot{\mathbb{Q}}_{\alpha}\right)_{\alpha<\omega_{2}}\right)$ such that for each $\alpha<\omega_{2}$,

(1) $\mathbb{P}_{\alpha+1}=\mathbb{P}_{\alpha} * \dot{\mathbb{Q}}_{\alpha}$ and $\Vdash_{\mathbb{P}_{\alpha}}\left|\dot{\mathbb{Q}}_{\alpha}\right|=\aleph_{1}$. (This guarantees that each $\mathbb{P}_{\beta}$ has a dense subset of size $\aleph_{1}$.) 
(2) For some $\mathbb{P}_{\alpha}$-name $\dot{\mathbf{T}}$ for an $\aleph_{1}$-oracle as in Lemma 3.3 a),

$$
\Vdash_{\mathbb{P}_{\alpha}} \text { "㐫 } \alpha \text { satisfies the } \dot{\mathbf{T}} \text {-chain condition", }
$$

so that $\mathbb{P}_{\alpha+1}$ satisfies the $\mathbf{S}$-chain condition. (This guarantees that all $\mathbb{P}_{\beta}$ satisfy the $\mathbf{S}$-chain condition.)

The forcing notion $\mathbb{P}_{\omega_{2}}$ then satisfies the $\mathbf{S}$-chain condition by Lemma $3.3 \mathrm{~b}$ ) and hence, by Lemma 3.4, in every $\mathbb{P}_{\omega_{2}}$-generic extension the ground model reals will form a non-meager, in fact a nowhere meager, subset of $\mathbb{R}$ of size $\aleph_{1}$.

By a theorem of Kuratowski [9], for any two perfect Polish spaces $X$ and $Y$ there are meager Borel sets $A \subseteq X$ and $B \subseteq Y$ so that $X \backslash A$ and $Y \backslash B$ are homeomorphic. Thus, if $\mathbb{R}$ has a a nowhere meager set of size $\aleph_{1}$ then every perfect Polish space has a nowhere meager set of size $\aleph_{1}$.

If in any $\mathbb{P}_{\omega_{2}}$-generic extension of the ground model, if $A$ and $B$ are nowhere meager subsets of size $\aleph_{1}$ of either the Urysohn space or of a given separable Banach space $X$, then there is $\alpha<\omega_{2}$ such that $A$ and $B$ are already elements of the corresponding $\mathbb{P}_{\alpha-}$ generic extension and they will be nowhere meager also in that intermediate model. Note that if $A$ and $B$ are nowhere meager subsets of a Banach space $X$, then they are dense in $X$ and therefore $X$ as a metric space can be reconstructed from either $A$ or $B$.

By using some suitable book-keeping we can make sure that for every $K>1$ and $A, B$ as above, there is some $\beta<\omega_{2}$ with $\alpha \leq \beta$ such that the forcing notion with the name $\dot{\mathbb{Q}}_{\beta}$ adds an $K$-bi-Lipschitz bijection between $A$ and $B$. The existence of such a forcing notion is guaranteed by Lemma 4.2 and by Lemma 4.3. For the book-keeping we use the facts that

(i) at every initial stage of the iteration we have $2^{\aleph_{1}}=\aleph_{2}$ and

(ii) there are only $\left(2^{\aleph_{0}}\right)^{\aleph_{1}}=2^{\aleph_{1}}$ separable metric spaces of size $\aleph_{1}$ (up to isometry).

It follows that in the $\mathbb{P}_{\omega_{2}}$-generic extension, any two sets $A$ and $B$ as above are almost isometric to each other.

Remark 5.2. The case $X=\mathbb{R}$ in Theorem 5.1 follows also from Burke's Theorem 1.7 in [5]: it is consistent that there is a nowhere meager subset of $\mathbb{R}$ of size $\aleph_{1}$ and any two such sets are order isomorphic via a restriction to $\mathbb{R}$ of an entire function with a first derivative arbitrarily close to 1 .

Theorem 5.3. If ZFC is consistent, then so is ZFC $+2^{\aleph_{0}}=\aleph_{2}+(\mathrm{a})+(\mathrm{b})+(\mathrm{c})$, where:

(a) There is an almost-isometry ultrahomogeneous and almost-isometry universal element in the class of all separable metric spaces of size $\aleph_{1}$.

(b) For every separable Banach space B there is a conditionally almost-isometry ultrahomogeneous and almost-isometry universal element in the class of subspaces of $B$ of size $\aleph_{1}$.

(c) For every finite-dimensional Banach space B, the property of being almostisometry universal in the class of subspaces of $B$ of cardinality $\aleph_{1}$ determines a space up to almost-isometry. 
Remark 5.4. Condition (c) easily fails for $\mathbb{U}$. Suppose $X \subseteq \mathbb{U}$ is universal and dense in $\mathbb{U}$, and let $X \cup\{x\}$ for a new point $x$ be so that $x$ has distance $\geq 1$ to any point in $X$. So both $X$ and $X \cup\{x\}$ are separable and almost-isometry universal, but they are not almost isometric to each other (and $X \cup\{x\}$ is not almost-isometry ultrahomogeneous). Similar examples exists in every separable Banach space which is isomorphic to one of its hyperplanes, e.g., the infinite dimensional Hilbert space.

Proof. We work in a model of set theory as in Theorem 5.1. To prove (a) fix a nowhere meager subset $Y \subseteq \mathbb{U}$ of cardinality $\aleph_{1}$. By Lemma 4.4, $Y$ is almost-isometry ultrahomogeneous. To prove that $Y$ is almost-isometry universal suppose that $X$ is a separable metric space of size $\aleph_{1}$. Since $X$ is isometric to a subspace of $\mathbb{U}$, we assume that $X$ is a subspace of $\mathbb{U}$. Now $X \cup Y$ is nowhere meager in $\mathbb{U}$ and of size $\aleph_{1}$, hence $Y$ and $X \cup Y$ are almost isometric. This implies that $X$ is almost isometrically embedded into $Y$.

The proof of (b) is similar to that of (a).

To prove (c) suppose $X$ is a finite-dimensional Banach space and that $A \subseteq X$ is of size $\aleph_{1}$ and is almost-isometry universal in the class of subspaces of $X$ of cardinality $\aleph_{1}$. We claim that $A$ is nowhere meager in $X$; the almost-isometry uniqueness of $A$ follows from this claim and $(\star)_{X}$.

Fix some set $B \subseteq X$ which is nowhere meager in $X$ and of cardinality $\aleph_{1}$ and fix, by the almost-isometry universality of $A$, some $K$-bi-Lipschitz embedding $f: B \rightarrow A$ for some $K>1$. Denote by $\hat{f}$ the continuous extension of $f$ to $X$. Also $\hat{f}$ is bi-Lipschitz.

Since $X$ is homeomorphic to $\mathbb{R}^{n}$ for some $n$, Brouwer's preservation of domain theorem applies to $X$, and therefore $\hat{f}$ is open; since $\hat{f}$ is bi-Lipschitz, $\hat{f}$ is also closed. Therefore ran $\hat{f}=\mathbb{R}^{n}$ by connectedness of $X$, and $\hat{f}$ is thus an autohomeomorphism of $X$. It follows that $\hat{f}[B]$ is a nowhere meager subset of $X$. Since $\hat{f}[B] \subseteq A, A$ is nowhere meager in $X$.

\section{Open problems}

Let us state the central open problem first:

Problem 6.1. Is it consistent to have an almost-isometry universal metric space in cardinality $\aleph_{1}<2^{\aleph_{0}}$ ?

We remark, again, that with any uncountable regular $\kappa>\aleph_{1}$ substituted for $\aleph_{1}$ in this problem, the answer is negative. The analogous problem for linearly ordered sets [12] and graphs and other relational structures [13, 10] have positive answers.

Problem 6.2. Is it consistent that there is a nowhere meager subset of $\mathbb{U}$ of cardinality $\aleph_{2}$ and that any two nowhere meager subsets of $\mathbb{U}$ of cardinality $\aleph_{2}$ are almost isometric?

A positive solution will solve positively also the next problem:

Problem 6.3. Is it consistent that $2^{\aleph_{0}}>\aleph_{2}$ and that there is an almost-isometry universal separable metric space of size $\aleph_{2}$ ?

Finally:

Problem 6.4. Is $(\star)_{\mathbb{U}}$ consistent with $2^{\aleph_{0}}>\aleph_{2}$ ? 


\section{Acknowledgements}

The research was supported by a German-Israeli Foundation grant number I-802195.6/2003. This note was written during a Research-in-Pairs stay at Mathematisches Forschungsinstitut Oberwolfach. We thank the referee for pointing out that Fact 2.2 holds for separable metric spaces, not just for perfect Polish spaces.

\section{References}

[1] U. Abraham, M. Rubin, and S. Shelah, On the consistency of some partition theorems for continuous colorings, and the structure of $\aleph_{1}$-dense real order types, Ann. Pure Appl. Logic 29 (1985), no. 2, 123-206.

[2] U. Avraham, S. Shelah, Martin's axiom does not imply that every two $\aleph_{1}$-dense sets of reals are isomorphic, Israel J. Math. 38 (1981), no. 1-2, 161-176.

[3] J. E. Baumgartner, All $\aleph_{1}$-dense sets of reals can be isomorphic, Fund. Math. 79 (1973), no. 2, 101-106.

[4] , Applications of the proper forcing axiom, Handbook of set-theoretic topology, K. Kunen and J. Vaughan eds., North-Holland, Amsterdam, 1984, 913-959.

[5] Maxim R. Burke, Entire functions mapping uncountable dense sets of reals onto each other monotonically, preprint.

[6] M. Džamonja, Club Guessing and the Universal Models, Notre Dame J. Formal Logic 46 (2005), no. $3,283-300$.

[7] M. Kojman and S. Shelah, Non existence of universal linear orders in many cardinalities, The Journal of Symbolic Logic, 57 (1992), no. 3, 857-891.

[8] _ Almost isometric embeddings between metric spaces, Israel J. Math. in press.

[9] K. Kuratowski, Topology, volume I, London, Panstwowe Wydawnictwo Naukowe, Warsaw, 1966.

[10] A. H. Mekler, Universal structures in power $\aleph_{1}$, J. Symbolic Logic 55 (1990), no. 2, 466-477.

[11] J. T. Moore, A five element basis for the uncountable linear orders, Ann. of Math. (2) 163 (2006), no. 2, 669-688.

[12] S. Shelah, Independence results, J. Symbolic Logic 45 (1980), no. 3, 563-573.

[13] _ On universal graphs without instances of CH, Ann. Pure Appl. Logic 26 (1984), no. 1, $75-87$.

[14] _ Proper and Improper Forcing, Perspectives in Mathematical Logic, Springer-Verlag, Berlin, Heidelberg, 1998.

[15] W. Sierpinski, Sur les types d'ordre des ensembles linaires, Fund. Math. 37 (1950), 253-264.

[16] B. Velickovic, Forcing axioms and stationary sets, Adv. Math. 94 (1992), no.2, 256-284.

[17] S. Todorcevic, Comparing the continuum with the first two uncountable cardinals, Dalla Chiara, Maria Luisa (ed.) et al., Logic and scientific methods, Volume one of the proceedings of the tenth international congress of logic, methodology and philosophy of science, Florence, Italy, August 19-25, 1995, Kluwer Academic Publishers. Synth. Libr. 259, 145-155 (1997).

II. Mathematisches Institut, Freie Universität Berlin, Arnimallee 3, 14195 Berlin, Germany and Boise State University, 1910 University Drive, Boise, ID 83725-1555, USA

E-mail address: geschke@math.fu-berlin.de

Department of Mathematics, Ben-Gurion University of the Negev, Beer Sheva, Israel E-mail address: kojman@math.bgu.ac.il 